\title{
Hexagonal vs. Rectilinear Grids for Explicit Finite Difference Schemes for the Two-dimensional Wave Equation
}

\author{
Brian Hamilton*1 and Stefan Bilbao ${ }^{1}$ \\ ${ }^{1}$ Acoustics Group, University of Edinburgh
}

To be presented at the 21st International Congress on Acoustics, Montréal, Canada, 2013

\begin{abstract}
Finite difference schemes for the 2-D wave equation operating on hexagonal grids and the accompanying numerical dispersion properties have received little attention in comparison to schemes operating on rectilinear grids. This paper considers the hexagonal tiling of the wavenumber plane in order to show that the hexagonal grid is a more natural choice to emulate the isotropy of the Laplacian operator and the wave equation. Performance of the 7-point scheme on a hexagonal grid is better than previously reported as long as the correct stability limit and tiling of the wavenumber plane are taken into account. Numerical dispersion is analysed as a function of temporal frequency to demonstrate directional cutoff frequencies. A comparison to 9-point compact explicit schemes on rectilinear grids is presented using metrics relevant to acoustical simulation. It is shown that the 7-point hexagonal scheme has better computational efficiency than parameterised 9-point compact explicit rectilinear schemes. A novel multiply-free 7-point hexagonal scheme is introduced and the 4-point scheme on a honeycomb grid is discussed.
\end{abstract}

*b.hamilton-2@sms.ed.ac.uk 


\section{INTRODUCTION}

The finite difference method can be used to make a numerical approximation to the solution of a partial differential equation (PDE). When applied to the second-order scalar wave equation [1] or equivalent firstorder vector equations [2], the method is more commonly known as the finite difference time domain (FDTD) method [3]. In this study, we are concerned with the 2-D scalar wave equation. One drawback of the FDTD method for the 2-D wave equation is numerical dispersion, which is unfavourable for acoustical simulations because it can produce audible artifacts due to mistuning of resonant modes and non-linear group delay [4]. Numerical dispersion is unavoidable but its severity depends on the particular choice of the finite difference operators in the scheme, and mostly on the approximation to the Laplacian operator and the spatial grid on which it operates (the same centered time difference operator is used in most explicit FDTD methods). Most FDTD research has focused on the use of rectilinear grids (most often square grids), on which the simplest second-order accurate FDTD scheme employs five points in space. In the 5-point scheme, numerical dispersion is worst for waves travelling in axial directions of the grid [4]. Second-order accurate 9-point schemes can better emulate the isotropy of the wave equation [4], but numerical dispersion is still significant over most of the simulated bandwidth. Aside from pre- and post-processing with frequency-warping techniques [5], the only way to reduce the numerical dispersion in a given scheme over some bandwidth of interest is to reduce the grid spacing and time step, but this incurs a greater computational cost. The computational cost of minimising numerical dispersion over some range of frequencies in second-order accurate explicit FDTD schemes for the 2 -D wave equation has been the subject of many studies [6, 7, 8]. Fourth-order accurate schemes do more to reduce numerical dispersion and can be achieved through implicit formulations [9], but the parallelism of explicit schemes is lost. Using wider spatial stencils in explicit schemes can also achieve higher-order accuracy $[10,11,12]$, but this can complicate the implementation of stable boundary conditions.

Another less employed strategy, and the focus of this paper, is to use the hexagonal spatial grid to approximate the Laplacian operator with seven points [13]. The hexagonal grid has been used in other finite differences approximations [14, 15, 16, 17, 18], but has not received much attention in FDTD literature aside from some studies [19, 3, 7, 20, 21]. It has received some attention in the Transmission Line Matrix (TLM) [22] and the Digital Waveguide Mesh (DWM) [23, 5, 24] literatures, both of which are scattering-based methods equivalent to the FDTD method at the passivity limit [7]. In the case of the 7-point hexagonal scheme, however, the more general FDTD method allows for a larger Courant number than the DWM/TLM formulation, resulting in less numerical dispersion [7]. It is well-known that 2-D signals on a hexagonal grid have spectra which are periodic as a hexagonal tiling [25]. This has been applied to finite difference schemes for other PDEs [18], but has yet to be correctly used to analyse the numerical dispersion in the wavenumbers of the 7-point FDTD scheme on a hexagonal grid. The purpose of this paper is two-fold: to analyse the numerical dispersion of the 7-point FDTD scheme on a hexagonal grid and to compare the 7-point hexagonal scheme to the 9-point rectilinear scheme in terms of computational efficiency. The paper is organised as follows. We introduce finite difference schemes for the 2-D wave equation, we present the numerical dispersion of the hexagonal scheme, and finally, we compare its computational efficiency to the 9-point rectilinear schemes.

\section{FINITE DIFFERENCE SCHEMES FOR 2-D WAVE EQUATION}

We are interested in the 2-D wave equation:

$$
\left(\frac{\partial^{2}}{\partial t^{2}}-c^{2} \Delta\right) u=0, \quad \Delta=\frac{\partial^{2}}{\partial x^{2}}+\frac{\partial^{2}}{\partial y^{2}} \equiv \frac{2}{3}\left(\frac{\partial^{2}}{\partial x_{1}^{2}}+\frac{\partial^{2}}{\partial x_{2}^{2}}+\frac{\partial^{2}}{\partial x_{3}^{2}}\right),
$$

where $c$ is the wave speed, $\Delta$ is the 2-D Laplacian operator, $t$ is time, and $u=u(t, x, y)$ is the solution we approximate. Boundary conditions will not be considered here, so $(x, y) \in \mathbb{R}^{2}$. In Eq. (1) we have rewritten the 2-D Laplacian in terms of three linearly dependent axes $x_{1}, x_{2}, x_{3}$ to simplify notation using a hexagonal spatial grid. The axes $x_{1}, x_{2}, x_{3}$ are represented by the unit vectors in $\mathbb{R}^{2}$ :

$$
\mathbf{x}_{1}=\left(\begin{array}{l}
1 \\
0
\end{array}\right), \quad \mathbf{x}_{2}=\left(\begin{array}{c}
-1 / 2 \\
\sqrt{3} / 2
\end{array}\right), \quad \mathbf{x}_{3}=\left(\begin{array}{c}
-1 / 2 \\
-\sqrt{3} / 2
\end{array}\right) .
$$




\section{Grids and Grid Functions}

To approximate our solution we must choose a spatial grid or lattice. ${ }^{1}$ A regular 2-D spatial grid is a collection of points defined as:

$$
\mathbf{G}_{h}=\left\{\mathbf{r}_{m_{1}, m_{2}}=\left(m_{1} \mathbf{x}_{1}+m_{2} \mathbf{x}_{\mathbf{2}}\right) h,\left(m_{1}, m_{2}\right) \in \mathbb{Z}^{2}\right\},
$$

where $\mathbf{x}_{1}, \mathbf{x}_{\mathbf{2}}$ are unit vectors that span $\mathbb{R}^{2}$ and $h$ is the grid spacing. The rectilinear (square) grid uses the unit vectors $\mathbf{x}_{1}=(1,0), \mathbf{x}_{2}=(0,1)$ and is shown in Fig. 1a. The density of points of the rectilinear spatial grid is $1 / h^{2}$. The hexagonal grid uses the unit vectors $\mathbf{x}_{1}, \mathbf{x}_{2}$ defined in Eq. (2) and is shown in Fig. 1b. We note that two interleaved hexagonal spatial grids can be overlaid on a rectilinear spatial grid with two grid spacings $h_{x}, h_{y}$ such that $h_{x}^{2}=3 h_{y}^{2}$ and $h=2 h_{x}$ [31]. ${ }^{2}$ The density of points of the hexagonal spatial grid is $2 / \sqrt{3} h^{2}$.

We define a grid function $u_{m_{1}, m_{2}}^{n}$ as a time series at each point on the spatial grid which approximates the continuous function $u(t, x, y)$ at time $t=n k$, where $k$ is the time step, and at the spatial position $(x, y)=\mathbf{r}_{m_{1}, m_{2}}$.

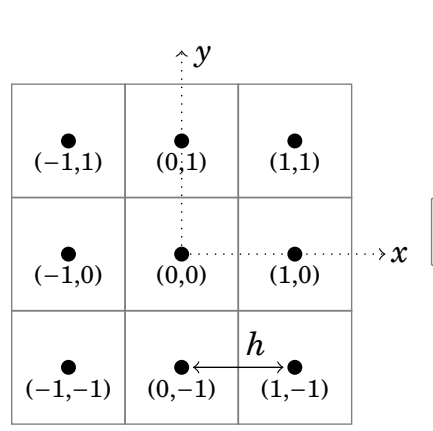

(A)

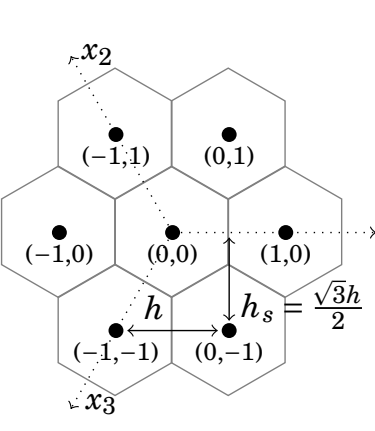

(B)

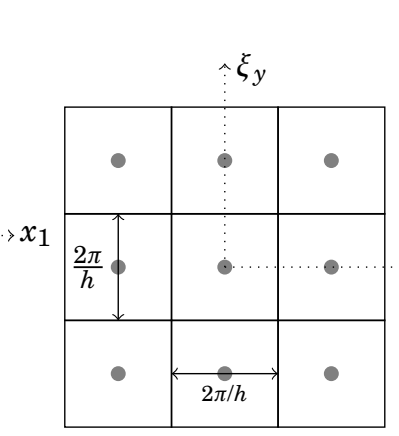

(C)

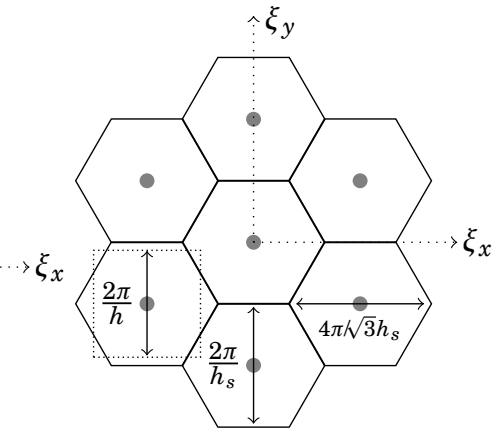

(D)

FIGURE 1: Grids (lattices) and their duals. (a) Rectilinear (square) grid. (b) Hexagonal grid. (c) Dual of square grid and wavenumber tiling. (d) Dual of hexagonal grid and wavenumber tiling.

\section{Tilings in Wavenumber Space}

Discrete functions on a 2-D spatial grid have Fourier transforms which are periodic in the spatial frequencies, or, wavenumbers [25]. This periodicity is a tiling of the wavenumber space which is given by the Voronoi tessellation of the reciprocal lattice (dual grid) of the direct lattice. There are many definitions of the reciprocal lattice [29, 25, 32], and we adopt a definition and approach similar to Hammond [32]. We define the vectors $\hat{\mathbf{x}}_{i}$ of the reciprocal lattice as the normal vectors to the lattice lines (lines connecting nearest neighbouring points on the grid) of the direct lattice scaled by the ratio $h_{s} / h$ where $h_{s}$ is the spatial period of the grid (known as the $d$-spacing in crystallography [32]). The points on the reciprocal lattice are multiplied by $2 \pi / h$ to represent the wavenumbers in units $\mathrm{rad} / \mathrm{m}$ :

$$
\boldsymbol{\xi}_{m_{1}, m_{2}}=2 \pi\left(m_{1} \hat{\mathbf{x}}_{\mathbf{1}}+m_{2} \hat{\mathbf{x}}_{\mathbf{2}}\right) / h \in \mathbb{R}^{2},\left(m_{1}, m_{2}\right) \in \mathbb{Z}^{2} .
$$

The wavenumbers $\boldsymbol{\xi}_{m_{1}, m_{2}}$ denote the centers of the translated copies of the wavenumber cell, known as the Brillouin zone in crystallography [32], which make up the wavenumber tiling of $\left(\xi_{x}, \xi_{y}\right) \in \mathbb{R}^{2}$.

\footnotetext{
${ }^{1}$ We use the terms grid and lattice interchangeably throughout this paper. The common use of the word 'grid' in the finite difference literature talks about a 'grid of points' [26], rather than a tessellation of space with 'grid cells' as in the finite element method literature [27]. In group theory, a lattice is a collection of points which can be generated by one set of lattice vectors [28]. In crystallography, a lattice can have more than one set of generating vectors, such as the 'honeycomb lattice' (the vertices of a hexagonal tiling) and the 'diamond cubic lattice' [29]. A crystal lattice is known as a 'Bravais lattice' when it is a lattice in the group theory sense [29]. Other finite difference texts [13] and the DWM literature [30], speak about nets or meshes, which are tilings of space by polygons. The finite difference approximation is made at the vertices of the tiling, which denote a lattice, so the 'triangular mesh' denotes the 'hexagonal lattice' and the 'hexagonal mesh' denotes the 'honeycomb lattice'. A spatial grid is a grid with units of space. A regular spatial grid is a spatial grid which has a single grid spacing. We also use the terms 'grid' and 'spatial grid' interchangeably.

${ }^{2}$ The same 7-point stencil considered in the following section is used by Bystrytskyi \& Moskal'kov on such a rectilinear grid [31]. Ultimately, this results in two uncoupled approximations, one on each interleaved grid (without considering coupling at boundaries).
} 
In the square lattice, the spatial period $h_{s}$ is simply $h$, the reciprocal lattice vectors are $\operatorname{simply} \hat{\mathbf{x}}_{1}=(1,0)$ and $\hat{\mathbf{x}}_{2}=(0,1)$, and the Voronoi tessellation is a square tiling of the wavenumbers made up of square cells with sides of length $2 \pi / h$, as shown in Fig. 1c. In the hexagonal lattice, the spatial period is $h_{s}=\sqrt{3} h / 2$, so the reciprocal lattice is given by the vectors:

$$
\hat{\mathbf{x}}_{1}=\left(\begin{array}{c}
1 \\
1 / \sqrt{3}
\end{array}\right), \quad \hat{\mathbf{x}}_{2}=\left(\begin{array}{c}
0 \\
2 / \sqrt{3}
\end{array}\right) .
$$

The dual of hexagonal grid and its wavenumber tiling are shown in Fig. 1d. Note that the distance between parallel sides of the hexagonal cell is $2 \pi / h_{s}$. A square cell with sides of length $2 \pi / h$ is inscribed in a hexagonal cell for comparison.

\section{Finite Difference Operators}

In the FDTD method, differential operators are approximated by finite difference operators. We start by defining the unit shift operators for our grid function:

$$
s_{t \pm} u_{m_{1}, m_{2}}^{n}=u_{m_{1}, m_{2}}^{n \pm 1}, \quad s_{x_{1} \pm} u_{m_{1}, m_{2}}^{n}=u_{m_{1} \pm 1, m_{2}}^{n}, \quad s_{x_{2} \pm} u_{m_{1}, m_{2}}^{n}=u_{m_{1}, m_{2} \pm 1}^{n}, \quad s_{x_{3} \pm}=s_{x_{1} \mp} s_{x_{2} \mp} .
$$

For the hexagonal grid, we have an extra shift operator, $s_{x_{3} \pm}$, along the $x_{3}$ axis for ease of analysis. Now we can build second-order finite difference operators as:

$$
\delta_{t t}=\left(s_{t-}+s_{t+}-2\right) / k^{2}, \quad \delta_{x_{i} x_{i}}=\left(s_{x_{i-}}+s_{x_{i}+}-2\right) / h^{2} .
$$

Using Taylor series expansions it is straightforward to show that these operators are second-order accurate approximations to the partial derivatives in Eq. (1). We can build a second-order accurate 5-point approximation to the Laplacian, $\delta_{\Delta \leftarrow:}$, on the rectilinear grid using the center point and its four nearest neighbours (NN); a 5-point approximation, $\delta_{\Delta::}$, using the center point and its four next nearest neighbours (NNN); and a secondorder accurate 9 -point approximation, $\delta_{\Delta \alpha: 3}$, as their linear combination with a free parameter $\alpha \in \mathbb{R}, \alpha \neq(0,1)$ :

$$
\delta_{\Delta: \bullet}=\delta_{x_{1} x_{1}}+\delta_{x_{2} x_{2}}, \quad \delta_{\Delta::}=\left(\left(s_{x_{1}-}+s_{x_{1}+}\right)\left(s_{x_{2}-}+s_{x_{2}+}\right)-4\right) / 2 h^{2}, \quad \delta_{\Delta \alpha: 3}=\alpha \delta_{\Delta:}+(1-\alpha) \delta_{\Delta::} .
$$

For the 9-point approximation, two special cases of interest are $\alpha=2 / 3$, from [13, 1], which results in isotropic error up to the fourth-order error term, and $\alpha=1 / 2$, which, when combined with $\delta_{t t}$ [33], can propagate waves with frequencies up to $\pi / k \mathrm{rad} / \mathrm{s}$ in every spatial direction [8]. This anisotropic scheme, dubbed the Interpolated Wideband (IWB) scheme, was found to be the most computationally efficient 9-point compact explicit scheme according to the efficiency measure devised by van Walstijn \& Kowalczyk [8].

On the hexagonal grid we employ seven points (center point and $6 \mathrm{NNs}$ ) to build a second-order accurate approximation to the Laplacian [13]:

$$
\delta_{\Delta \%}=\frac{2}{3}\left(\delta_{x_{1} x_{1}}+\delta_{x_{2} x_{2}}+\delta_{x_{3} x_{3}}\right)=\Delta+\frac{h^{2}}{16} \Delta^{2}+O\left(h^{4}\right) .
$$

The series expansion of Eq. (9) shows that $\delta_{\Delta *}$ is isotropic to the fourth-order in the grid spacing $h$ [13].

\section{Explicit Finite Difference Schemes}

We can now use the following difference equation to approximate a solution to Eq. (1):

$$
\left(\delta_{t t}-c^{2} \delta_{\Delta}\right) u_{m_{1}, m_{2}}^{n}=0,
$$

where $\delta_{\Delta}$ is some unspecified approximation to the Laplacian on a regular grid. On the rectilinear grid we use $\delta_{\Delta \alpha_{: \#}}$ and we have the explicit update equation:

$$
\begin{aligned}
& u_{m_{1}, m_{2}}^{n+1}=\lambda^{2} \alpha\left(u_{m_{1}+1, m_{2}}^{n}+u_{m_{1}-1, m_{2}}^{n}+u_{m_{1}, m_{2}+1}^{n}+u_{m_{1}, m_{2}-1}^{n}\right) \\
& \quad+\lambda^{2} \frac{(1-\alpha)}{2}\left(u_{m_{1}+1, m_{2}+1}^{n}+u_{m_{1}+1, m_{2}-1}^{n}+u_{m_{1}-1, m_{2}+1}^{n}+u_{m_{1}-1, m_{2}-1}^{n}\right)+2\left(1-\lambda^{2}(\alpha+1)\right) u_{m_{1}, m_{2}}^{n}-u_{m_{1}, m_{2}}^{n-1}
\end{aligned}
$$


where $\lambda=c k / h$ is the Courant number, which is the ratio between the time step and the grid spacing for a given wave speed $c$. On the hexagonal grid we use $\delta_{\Delta \%}$ and the explicit update is:

$$
u_{m_{1}, m_{2}}^{n+1}=\lambda^{2} \frac{2}{3}\left(u_{m_{1}+1, m_{2}}^{n}+u_{m_{1}-1, m_{2}}^{n}+u_{m_{1}, m_{2}+1}^{n}+u_{m_{1}, m_{2}-1}^{n}+u_{m_{1}+1, m_{2}+1}^{n}+u_{m_{1}-1, m_{2}-1}^{n}\right)+\left(2-4 \lambda^{2}\right) u_{m_{1}, m_{2}}^{n}-u_{m_{1}, m_{2}}^{n-1} .
$$

For a given scheme, $\lambda$ has a maximum value for which numerical stability is guaranteed [26].

\section{Stability Conditions}

Stability conditions are determined by finding the maximum value of $\lambda$ such that no exponentially-growing plane wave solutions of the form $u_{m_{1}, m_{2}}^{n}=e^{j\left(\omega n k+\left(\xi_{x}, \xi_{y}\right) \cdot\left(m_{1} \mathbf{x}_{1}+m_{2} \mathbf{x}_{2}\right) h\right)},\left(\omega, \xi_{x}, \xi_{y}\right) \in \mathbb{C}^{3},\left(n, m_{1}, m_{2}\right) \in \mathbb{Z}^{3}$ are permitted in the numerical scheme [26]. In general, the stability condition is completely determined by considering plane wave solutions with frequencies $\omega \in[0, \pi]$ and real wavenumbers $\xi_{x}, \xi_{y}$ in one wavenumber cell of the grid, since the Fourier symbol of $\delta_{\Delta}$ is periodic in the wavenumbers. In the case of the rectilinear grid, the stability condition is found at the corners of the square wavenumber cell because the Fourier symbol of $\delta_{\Delta \alpha}:: 3$ is multilinear in $\xi_{x}, \xi_{y}$ [33]. The stability condition for the 9-point explicit scheme is [33]:

$$
\alpha \geq 0, \quad \lambda \leq \min (1,1 / \sqrt{2 \alpha}) .
$$

The stability condition for the hexagonal case requires more care because multilinearity is lost in the Fourier symbol of $\delta_{\Delta *:}$ and because the region to be considered is a hexagon. ${ }^{3}$ Consequently, many studies have given the wrong stability limits [31,21], ${ }^{4}$ while other studies have used a TLM/DWM formulation [22, 23, 5], which employs the Courant number at a lower passivity limit [7]: $\lambda=\sqrt{1 / 2} .5$ The correct stability condition for the 7-point hexagonal scheme, given by Liu [19] for the equivalent FDTD scheme for Maxwell's equations, and by Fabero \& Bautista [34] and Bilbao [7] for the wave equation scheme, is:

$$
\lambda \leq \sqrt{2 / 3} .
$$

Notice in Fig. $1 \mathrm{~d}$ that considering $\xi_{x}, \xi_{y} \in[-\pi / h, \pi / h]$ is not sufficient to cover the entire hexagonal wavenumber cell. In fact, the bound in Eq. (14) is achieved at the corners of the hexagon. Searching over a larger area will inevitably encompass the corners, so considering the hexagonal tiling of the wavenumbers is not necessary in determining the stability condition. However, considering such a tiling is necessary to fully analyse the numerical dispersion of the scheme, as we will see in the next section.

\section{NUMERICAL DISPERSION}

The conditions for which plane waves of the form $u=e^{j\left(\omega t+\xi_{x} x+\xi_{y} y\right)}$ are solutions to the 2-D wave equation are given by the well-known dispersion relation:

$$
\omega^{2}=c^{2}|\xi|^{2},
$$

where $\boldsymbol{\xi}=\left(\xi_{x}, \xi_{y}\right)$. The phase velocity, or wave speed, for $|\xi|>0$ is then $\omega /|\xi|=c$. The finite-difference scheme approximates Eq. (15) in the frequency domain (space and time), and the numerical dispersion relation is:

$$
\mathscr{D}_{t t}(\omega)=c^{2} \mathscr{D}_{\Delta}(\xi)
$$

for some $\mathscr{D}_{t t}: \mathbb{C} \rightarrow \mathbb{C}, \mathscr{D}_{\Delta}: \mathbb{C}^{2} \rightarrow \mathbb{C}$ which are the Fourier symbols of the finite difference operators of the scheme. In practice, we only consider the real frequencies and wavenumbers, but in general, the mapping is complex. For the 7-point hexagonal scheme, we can write these operators as:

$$
\mathscr{D}_{t t}(\omega)=-\frac{4}{k^{2}} \sin ^{2}(\omega k / 2), \quad \mathscr{D}_{\Delta \%}(\boldsymbol{\xi})=-\frac{8}{3 h^{2}} \sum_{i=1}^{3} \sin ^{2}\left(\boldsymbol{\xi} \cdot \mathbf{x}_{i} h / 2\right) .
$$

\footnotetext{
${ }^{3}$ The operator $\delta_{\Delta . \%}$ can be viewed as a 2-D filter on the hexagonal grid [25].

${ }^{4}$ Bystrytskyi \& Moskal'kov arrived at $\lambda \leq \sqrt{3} / 2$ for the same scheme [31] but this is slightly higher than Eq. (14) and leads to instability.

${ }^{5}$ This was incorrectly given as the stability condition by Fei et al. [21]. It is worth noting that their FDTD scheme is equivalent to that of Liu [19], just reformulated for the transverse electric modes.
} 
For the 9-point rectilinear scheme, only $\mathscr{D}_{\Delta}$ changes:

$$
\mathscr{D}_{\Delta \alpha=3}(\xi)=-\frac{4}{h^{2}}\left(\sin ^{2}\left(\xi_{x} h / 2\right)+\sin ^{2}\left(\xi_{y} h / 2\right)-2(1-\alpha) \sin ^{2}\left(\xi_{x} h / 2\right) \sin ^{2}\left(\xi_{y} h / 2\right)\right) .
$$

To make $\mathscr{D}_{t t}$ injective we consider the real wavenumbers $\xi \in \mathbb{B}$, where $\mathbb{B}$ is the wavenumber cell of the grid, and we consider only the positive square root. If the stability conditions are satisfied then $\mathscr{D}_{t t}^{-1}\left(c^{2} \mathscr{D}_{\Delta}(\xi)\right)$ maps from $\mathbb{B} \rightarrow[0, \pi / k]$. We can then write the phase velocity as a function of the wavenumber $(|\xi|>0)$ :

$$
v(\xi)=\frac{\omega(\boldsymbol{\xi})}{|\boldsymbol{\xi}|}, \quad \omega(\boldsymbol{\xi})=\mathscr{D}_{t t}^{-1}\left(c^{2} \mathscr{D}_{\Delta}(\boldsymbol{\xi})\right) .
$$

This has been the traditional way to analyse numerical wave speed error in FDTD schemes across all the wavenumbers [4], but $v(\xi)$ has not, to our knowledge, been examined on the correct domain $\mathbb{B}$ for the hexagonal scheme. We plot the dispersion of the hexagonal scheme in Fig. 2 for both the stable and passive schemes on the appropriate domain $\mathbb{B}$ for the hexagonal grid. If one assumes a square wavenumber cell, either some of the wavenumbers will be missing, or the cell will contain aliased wavenumbers, which will give an incorrect numerical wave speed without adjusting the denominator in Eq. (19) accordingly. It is less apparent in Fig. 2a than in Fig. 2b, but the 7-point scheme is isotropic (to the fourth-order error term) due to the result in Eq. (9). ${ }^{6}$

Van Walstijn \& Kowalczyk [8] realised that another useful representation of the numerical dispersion is given by translating Eq. (19) back to an angle and temporal frequency via the dispersion relation and generating polar plots where the polar radius represents the temporal frequency $\omega$, and the polar angle represents the angle of propagation $\theta$ for $\xi=(|\xi|, \theta)$. In other words, we write the numerical wave speed as $v(\omega(|\xi|, \theta), \theta)$ instead of $v(\xi)$. Polar plots generated from $v(\omega(|\xi|, \theta), \theta)$ happen to be more useful for audio simulations since we hear pressure variations over time. Using this representation, the error in the wave speed for the two hexagonal schemes is plotted in Figs. 3a and 3b.

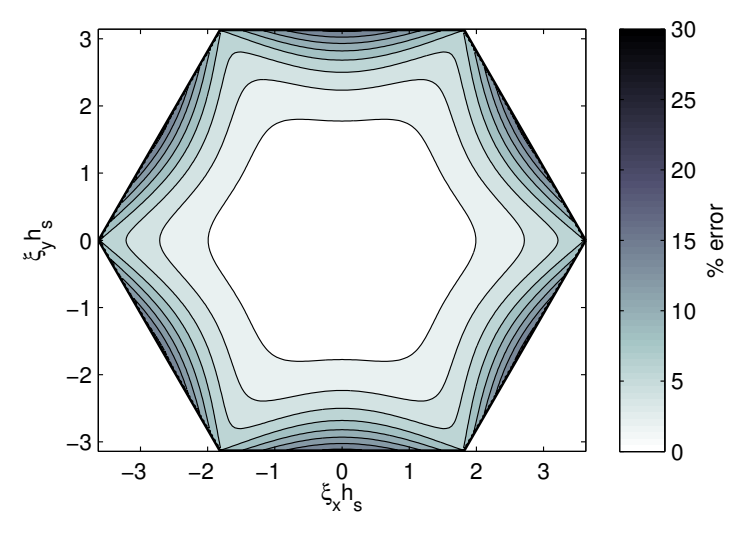

(A) $\lambda=\sqrt{2 / 3}$

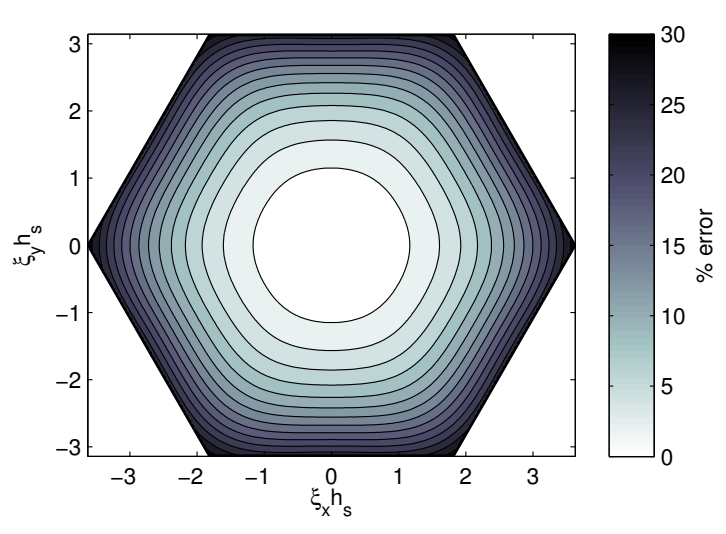

(B) $\lambda=\sqrt{1 / 2}$

FIGURE 2: Relative percent error in $v(\xi)$ for hexagonal scheme in wavenumber cell, $2 \%$ error contours

These polar plots also show the cutoff frequency in each direction. The cutoff frequency for a fixed angle $\theta$ is the maximum frequency $\omega$ of a plane wave that will propagate in the scheme. ${ }^{7}$ We note that the stable 7-point scheme has a global cutoff frequency at $\pi / k \mathrm{rad} / \mathrm{s}$, and the minimum directional cutoff frequency is $\omega=2 \sin ^{-1}(2 \sqrt{2} / 3) / k \approx 0.784 \pi / k \mathrm{rad} / \mathrm{s}$. The passive 7-point scheme has a minimum cutoff frequency of $\omega=2 \sin ^{-1}(\sqrt{2 / 3}) / k \approx 0.608 \pi / k \mathrm{rad} / \mathrm{s}$ and a maximum global cutoff at $\omega=2 \pi / 3 k \mathrm{rad} / \mathrm{s}$. We also plot the wave speed error for the two special cases of the 9-point rectilinear scheme in Figs. 3c and 3d (the 5-point scheme can be found elsewhere [8]). A quick comparison shows that the 7-point stable scheme has a $2 \%$ error at just over $0.5 \pi / k$ for the worst direction (along the diagonals), while the IWB scheme reaches $2 \%$ error at about $0.4 \pi / k$ for its worst direction (along the diagonals). Indeed, the IWB scheme is exact for plane waves travelling

\footnotetext{
${ }^{6}$ The Taylor series only tells us that the scheme is isotropic in the neighbourhood of $\xi h=0$.

${ }^{7}$ In fact, frequencies higher than the cutoff can propagate over short distances, as shown by Schneider [35], but this phenomenon is negligible.
} 


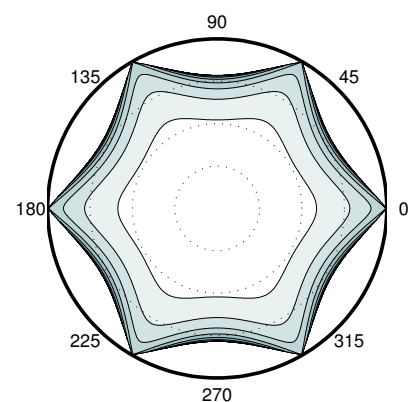

(A) 7-point, $\lambda=\sqrt{2 / 3}$

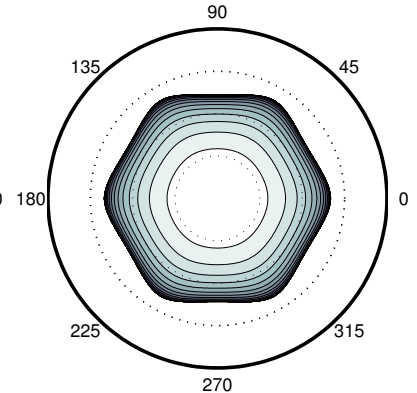

(B) 7-point, $\lambda=\sqrt{1 / 2}$

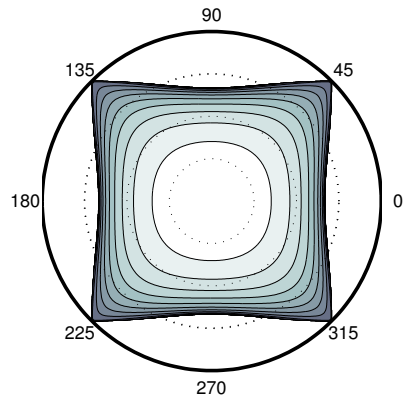

(C) 9-point, $\alpha=2 / 3, \lambda=\sqrt{3} / 2$

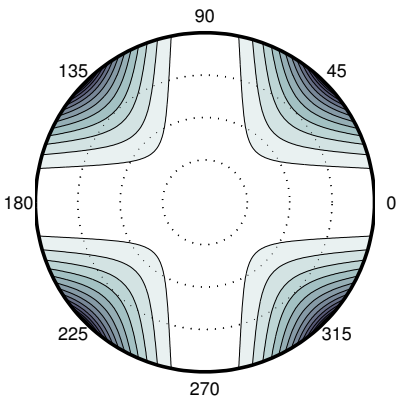

(D) 9-point, $\alpha=1 / 2, \lambda=1$

FIGURE 3: Relative percent error in $v(\omega(|\xi|, \theta), \theta)$ for 7-point hexagonal scheme and 9-point rectilinear schemes, $2 \%$ error contours, radial axis is $\omega \in(0, \pi / k] \mathrm{rad} / \mathrm{s}$. Colour axis is the same as in Fig. 2.

along the axes, but we consider the worst-case direction to be most critical since the numerical wave speed error should be acceptable in any given direction.

\section{Computational EFFiCiEnCY}

The computational cost of a finite difference scheme can be measured in terms of the computational density of the scheme, defined as the number of grid points that need to be updated per unit area per unit time. The computational density for the rectilinear scheme, $\rho_{R}$, with grid spacing $h_{R}$ and time step $k_{R}$, and the computational density for the hexagonal scheme, $\rho_{H}$, with $h_{H}, k_{H}$, can be written as:

$$
\rho_{R}=\left(h_{R}^{2} k_{R}\right)^{-1}, \quad \rho_{H}=(2 / \sqrt{3})\left(h_{H}^{2} k_{H}\right)^{-1} .
$$

We compare just the IWB scheme $\left(\alpha=1 / 2, \lambda_{R}=1\right)$ to the 7-point hexagonal scheme $\left(\lambda_{H}=\sqrt{2 / 3}\right)$ since it was found to be the most efficient of the 9-point compact explicit family of schemes [8]. Fixing the time step such that $k_{R}=k_{H}=k$, we plot the dispersion along the worst direction for each scheme in Fig. 4a. We see that the hexagonal scheme has less dispersion than the IWB scheme up to about $0.73 \pi / k \mathrm{rad} / \mathrm{s}$, but above that frequency, the IWB has less dispersion and the 7-point scheme cuts off. However, with $k_{R}=k_{H}$ the computational density of the 7-point scheme is less $\left(\rho_{H}=4 / 3 \sqrt{3} \rho_{R} \approx 0.77 \rho_{R}\right)$, so we could afford to reduce the time step and use more points. This makes it difficult to compare the two schemes in terms of computational efficiency, so we employ the relative efficiency measure adopted by van Walstijn \& Kowalczyk [8].

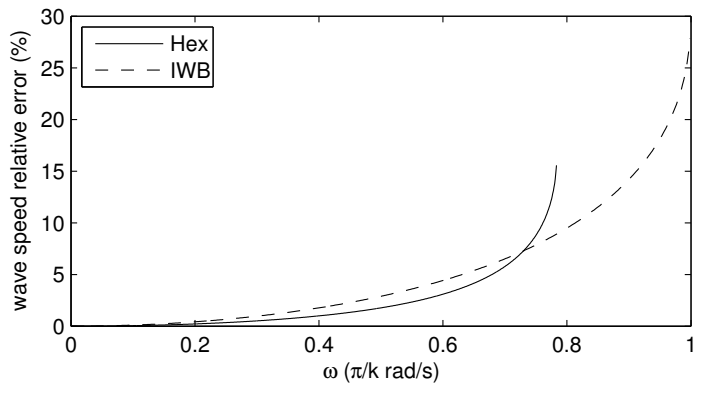

(A)

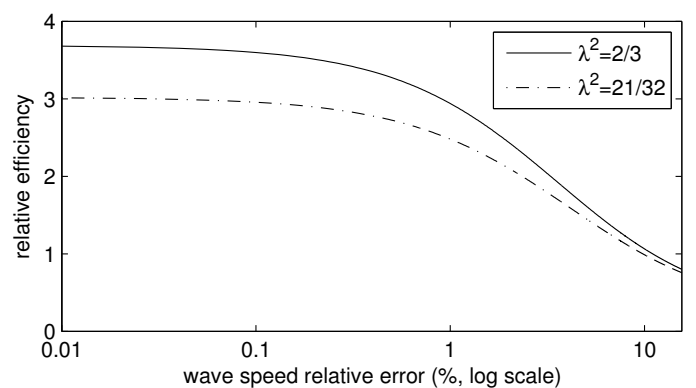

(B)

FIGURE 4: (a) Relative error in $v(\omega(|\xi|, \theta), \theta)$ along worst direction $\theta$ for 7-point hexagonal scheme and IWB scheme. (b) Relative efficiency of 7-point hexagonal scheme over IWB scheme.

The relative efficiency measures how well a scheme maintains the wave speed error below some threshold up to some critical frequency relative to another scheme. It reveals the increase or decrease in the computational density, relative to another scheme, required to achieve that threshold up to some critical frequency, 
since reducing the time step is the only way to reduce the wave speed error at a given temporal frequency in a scheme already at the stability limit. It is important to note that this measure is independent of the critical frequency [8]. The IWB scheme was found to be four times more efficient than the standard 5-point rectilinear scheme at every critical error using this measure [8]. The relative efficiency of the hexagonal scheme over the IWB scheme is plotted in Fig. $4 \mathrm{~b}$, from $0.01 \%$ to $15 \%$ error. We see that the 7-point hexagonal is more efficient than the IWB scheme when $10 \%$ error or less is considered to be the critical error. When less than $1 \%$ error is considered, the 7-point scheme has anywhere from 3 to 3.7 times the computational efficiency of the IWB scheme. This translates to 12 to 15 times the efficiency of the 5-point scheme, and better efficiency than all other 9-point explicit schemes. We can also consider the specific arithmetic operations required at each point and time step, but the 7-point scheme requires fewer additions (seven vs nine) and the same number of multiplications (two) so it remains more efficient. Finally, since the hexagonal scheme uses fewer neighbouring points, the memory bandwidth is also lower.

\section{Multiply-free Implementation}

If the IWB scheme is implemented in a fixed point binary representation multiplications can be replaced with bit shifts. We can use a similar trick for the hexagonal scheme with $\lambda^{2}=21 / 32 \approx 2 / 3$. The multiplications in Eq. (12) can then be implemented with $2 \lambda^{2} / 3=2^{-2}+2^{-3}+2^{-4}$ and $2-4 \lambda^{2}=-\left(2^{-1}+2^{-3}\right)$. This multiply-free scheme is suboptimal, yet still more efficient than the IWB scheme below $10 \%$ error, as seen by the dashed line in Fig. 4b, even with three extra additions.

\section{Honeycomb Grid}

Without going into much detail, we note that one can use two mirror-image first-order accurate 4-point approximations to the Laplacian on a honeycomb grid [13] to build a second-order accurate FDTD scheme for the 2-D wave equation [7] (known as the 'hexagonal DWM' from [30]). Considering two time steps, this scheme at the stability limit can be reduced to two uncoupled (not considering boundaries) 7-point schemes on hexagonal grids at the stability limit with grid spacing $h^{\prime}=\sqrt{3} h$, where $h$ is the grid spacing on the honeycomb grid [7]. The 7-point hexagonal scheme has a relative efficiency of four over the 4-point honeycomb scheme because the honeycomb scheme uses twice the number of grid points (two uncoupled hexagonal grids) and requires twice the number of time steps.

\section{CONClusion}

In this paper, we have analysed the 7-point hexagonal FDTD scheme for the 2-D wave equation in terms of its numerical dispersion over the full hexagonal wavenumber cell and in terms of computational costs relative to the 9-point compact explicit rectilinear schemes. It was shown that when less than $10 \%$ error in the wave speed is desired at some critical frequency the 7-point scheme is more efficient than all 9-point compact explicit schemes. A new, suboptimal multiply-free implementation of the 7-point scheme was introduced, which is better than optimal multiply-free 9-point rectilinear schemes. The relationship of the 7-point scheme to the 4-point FDTD scheme on a honeycomb grid was discussed.

We have only used one efficiency measure, but others could be considered. For example, instead of choosing a critical error for one frequency, we can consider the integrated wave speed error over a range of frequencies, or the rate of change of the wave speed error. Perceptual tests will be conducted in future work to determine what efficiency measure is most suitable for acoustical simulations. Other future work will include establishing stable frequency-dependent and absorbing boundary conditions for the hexagonal scheme, and examining higher-order schemes on the hexagonal grid.

\section{Acknowledgements}

This work was supported by the European Research Council, under grant StG-2011-279068-NESS, and by the Natural Sciences and Engineering Research Council of Canada. 


\section{REFERENCES}

[1] G. Forsythe and W. Wasow, Finite-difference methods for partial differential equations (New York: Wiley) (1960).

[2] K. Yee, "Numerical solution of initial boundary value problems involving Maxwell's equations in isotropic media", IEEE Trans. Antennas and Propagation 14, 302-307 (1966).

[3] A. Taflove and S. Hagness, Computational electrodynamics, volume 160 (Artech House London) (2000).

[4] L. Trefethen, "Group velocity in finite difference schemes", SIAM review 24, 113-136 (1982).

[5] L. Savioja and V. Valimaki, "Reducing the dispersion error in the digital waveguide mesh using interpolation and frequency-warping techniques”, IEEE Trans. Acoustics, Speech, and Signal Processing 8, 184-194 (2000).

[6] F. Fontana and D. Rocchesso, "Signal-theoretic characterization of waveguide mesh geometries for models of twodimensional wave propagation in elastic media", IEEE Trans. Acoustics, Speech, and Signal Processing 9, 152-161 (2001).

[7] S. Bilbao, Wave and scattering methods for numerical simulation (Wiley) (2004).

[8] M. van Walstijn and K. Kowalczyk, "On the numerical solution of the 2D wave equation with compact FDTD schemes", in Proc. Digital Audio Effects (DAFx), 205-212 (Espoo, Finland) (2008).

[9] G. Fairweather and A. Mitchell, "A high accuracy alternating direction method for the wave equation", IMA J. Applied Mathematics 1, 309-316 (1965).

[10] G. Shubin and J. Bell, "A modified equation approach to constructing fourth order methods for acoustic wave propagation", SIAM J. Scientific and Statistical Computing 8, 135-151 (1987).

[11] G. Cohen and P. Joly, "Description and analysis of schemes, fourth-order in time and space, for 1-D and 2-D acoustic equations", in Society of Exploration Geophysicists Annual Meeting (1987).

[12] S. Bilbao and B. Hamilton, "Construction and optimization techniques for high order schemes for the two-dimensional wave equation", in Proc. 21st International Congress on Acoustics (Montréal, Canada) (2013).

[13] L. Kantorovich and V. Krylov, Approximate methods of higher analysis. Translated by Curtis D. Benster (Interscience Publishers) (1958).

[14] R. Sadourny and P. Morel, "A finite-difference approximation of the primitive equations for a hexagonal grid on a plane”, Monthly Weather Review 97, 439-445 (1969).

[15] K. Pruess and F. Bodvarsson, "A seven-point finite difference method for improved grid orientation performance in pattern steamfloods", in SPE Reservoir Simulation Symposium (1983).

[16] M. Woodward and F. Muir, "Hexagonal finite difference operators and 3-d wave equation migration", Stanford Exploration Project 38, 195-206 (1984).

[17] D. Zingg and H. Lomax, "Finite-difference schemes on regular triangular grids", J. Computational Physics 108, 306313 (1993).

[18] S. Ničkovic, M. Gavrilov, and I. Tošic, "Geostrophic adjustment on hexagonal grids", Monthly Weather Review 130, 668-683 (2002).

[19] Y. Liu, "Fourier analysis of numerical algorithms for the Maxwell equations", J. Computational Physics 124, 396-416 (1996).

[20] J. Keranen, E. Koljonen, T. Tarhasaari, and L. Kettunen, "Effect of cell type on convergence of wave propagation schemes", IEEE Trans. Magnetics 40, 1452-1455 (2004).

[21] X. Fei, T. Xiaohong, and Z. Xianjing, "The construction of low-dispersive FDTD on hexagon”, IEEE Trans. Antennas and Propagation 53, 3697-3703 (2005).

[22] N. Simons, A. Sebak, and Y. Antar, “Two dimensional hexagonal TLM node and velocity error correction", in IEEE Antennas and Propagation Society International Symposium, 902-905 (1991).

[23] F. Fontana and D. Rocchesso, "A new formulation of the 2D-waveguide mesh for percussion instruments", in Proceedings of the XI Colloqium on Musical Informatics (Bologna, Italy) (1995).

[24] H. Hachabiboglu, B. Gunel, and A. Kondoz, "On the accuracy of first-order numerical derivatives in multidimensional digital waveguide mesh topologies”, IEEE Signal Processing Letters 15, 9-12 (2008).

[25] D. Dudgeon and R. Mersereau, "Multidimensional digital signal processing", Englewood Cliffs: Prentice-Hall 1 (1984).

[26] J. Strikwerda, Finite difference schemes and partial differential equations (Society for Industrial Mathematics) (2004).

[27] J. Thompson, B. Soni, and N. Weatherill, Handbook of grid generation (CRC Press) (1998).

[28] J. Conway and N. Sloane, Sphere packings, lattices and groups, volume 290 (Springer) (1998).

[29] N. Ashcroft and N. Mermin, Solid State Physics (Saunders College, Philadelphia) (1976).

[30] S. van Duyne and J. O. Smith III, "The 3D tetrahedral digital waveguide mesh with musical applications", in Proc. Int. Computer Music Conf. (ICMC), 9-16 (Hong Kong) (1996).

[31] M. Bystrytskyi and M. Moskal'kov, "The difference laplace operator on a seven-point nonorthogonal pattern of a rectangular grid and its spectral properties”, J. Mathematical Sciences 104, 1593-1598 (2001).

[32] C. Hammond, The Basics of Crystallography and Diffraction (Oxford University Press, USA) (2009).

[33] S. Bilbao, "Parameterized families of finite difference schemes for the wave equation", Numerical Methods for Partial Differential Equations 20, 463-480 (2004).

[34] J. Fabero, A. Bautista, and L. Casasús, "An explicit finite differences scheme over hexagonal tessellation", Applied Mathematics Letters 14, 593-598 (2001).

[35] J. Schneider and C. Wagner, "FDTD dispersion revisited: Faster-than-light propagation", IEEE Microwave and Guided Wave Letters 9, 54-56 (1999). 